\title{
Androgen Deprivation Therapy for Patients with Androgen-Receptor-Positive Metastatic Salivary Duct Carcinoma: A Case Report and Review of the Literature
}

\author{
Yongfei You (DD' \\ Panpan Wang' \\ Xi Wan ${ }^{2}$ \\ Liping $X u^{3}$ \\ Yi Gong ${ }^{4}$ \\ Weihua Zhang' \\ 'Department of Oncology, The First \\ Hospital of Nanchang, Nanchang, Jiangxi, \\ 330008, People's Republic of China; \\ ${ }^{2}$ Operating Room, The First Hospital of \\ Nanchang, Nanchang, Jiangxi, 330008, \\ People's Republic of China; ${ }^{3}$ Department \\ of Pathology, The First Hospital of \\ Nanchang, Nanchang, Jiangxi, 330008 , \\ People's Republic of China; ${ }^{4}$ Department \\ of Imaging, The First Hospital of \\ Nanchang, Nanchang, Jiangxi, 330008, \\ People's Republic of China
}

\begin{abstract}
Salivary duct carcinoma (SDC) is a rare and aggressive malignancy with high rates of recurrence and metastasis. There are no standard treatment options available for patients with recurrence and metastases. The case of a 61-year-old male with SDC of the left parotid gland is presented in this study. The results revealed that the patient's tumour had strong positive staining for androgen receptor (AR) expression, mutations in HRAS and PIK3CA but not in other related genes, and no gene amplification of HER-2. After the primary therapy of parotidectomy with neck dissection and postoperative radiation, bone metastases were found in the ribs, pelvis and spine. Androgen deprivation therapy (ADT) involving combined androgen blockade $(\mathrm{CAB})$ was effective as the first-line therapy for the patient's metastases and resulted in a progression-free survival (PFS) of over 7 months to date. In conclusion, androgen deprivation therapy is recommended for patients with recurrent or metastatic SDC positive for androgen receptor expression.
\end{abstract}

Keywords: salivary duct carcinoma, androgen receptor, androgen deprivation therapy, case report

\section{Introduction}

Salivary duct carcinoma (SDC) is a rare and aggressive tumour with 21 primary subtypes identified in the latest World Health Organization classification. SDC has a high rate of recurrence and metastasis. The majority of SDCs arise from the parotid gland. The standard treatments for SDC include total parotidectomy and ipsilateral neck dissection followed by postoperative radiation therapy with or without chemotherapy; however, SDC has a dismal prognosis. There are no standard treatment options available for patients with recurrence and metastasis. Multiple therapeutic targets have been identified, including the AR, and reports have shown that androgen deprivation therapy (ADT) might be effective. This case study reviewed the clinical data of a patient with SDC in the left parotid gland and discussed the relevant literature.

\section{Case Report}

A 61-year-old male presented with backache for 3 months. A soybean-sized mass accompanied by pain and numbness was found on the front and back of the left ear in 
October 2015, the left corner of the mouth was skewed and the left eye was partially closed. The patient was diagnosed with facial paralysis at the local hospital and his symptoms did not respond to acupuncture treatment. The mass on the patient's ear had grown to the size of a pigeon egg by 2017. The swelling, pain, numbness, skewing of the left corner of the mouth, and the left eye insufficiency were also aggravated. There was no fever or weight loss during the course of the disease. The patient had no history of tumours or autoimmune diseases and had no family history of tumours. A physical examination revealed several slightly enlarged lymph nodes under the left ear and in the jaw. The largest node was $1.5 \mathrm{~cm}$ in diameter and had a tough texture, good mobility and no tenderness. A hard, slightly tender mass approximately $2 \times 1.8 \mathrm{~cm}$ in size was noted below the front of the left ear with poor mobility, no redness or swelling of the local skin, and a low skin temperature. Computer tomography $(\mathrm{CT})$ of the parotid gland identified a mixed tumour that had undergone malignant transformation (Figure 1A and B). A fine-needle aspiration examination of the left parotid gland revealed that the tumour was of epithelial origin. The diagnosis of reactive lymphoid tissue hyperplasia was determined by a left ear lymph node puncture smear. No distant metastases were found in other organs. The clinical stage was estimated to be T4aN0M0.
The primary surgical treatment consisted of a total parotidectomy conserving the facial nerve with a modified ipsilateral radical neck dissection preserving the branches of the temporal and facial trunk and removing part of the zygomatic and buccal branches of the facial nerve. Post-operative pathology indicated that the patient had duct carcinoma of the parotid gland (Figure 1C) with invasion to surrounding soft tissues. Tumour thrombus and nerve invasion could be seen. The pathologist identified twenty-one lymph nodes, consisting of parotid glandadjacent lymph nodes (1/6), submandibular and upper deep cervical lymph nodes $(0 / 14)$ and cervical lymph nodes (0/1). On immunohistochemistry, the tumour demonstrated strong or weak positive staining for $\mathrm{CK}$, CK7 (weak), CK8/18, EMA, Cam5.2, CK (high) and Ki67 (10\%), and was negative for CK20, CEA, Vimentin, S-100, Calponin, CD117, P63 and Dog-1. The final pathological staging was pT4aN1M0. Three weeks after surgery, postoperative radiation therapy (66 Gy; 2.2 Gy once a day, five days a week) was applied to the surgical bed and the left neck area due to the aggressive nature of the tumour. No tumour recurrence or metastasis was found at followup until May 2020, when the patient's serum CA724 levels were found to be greater than $300 \mathrm{U} / \mathrm{mL}$. Emission computed tomography revealed multiple bone metastases
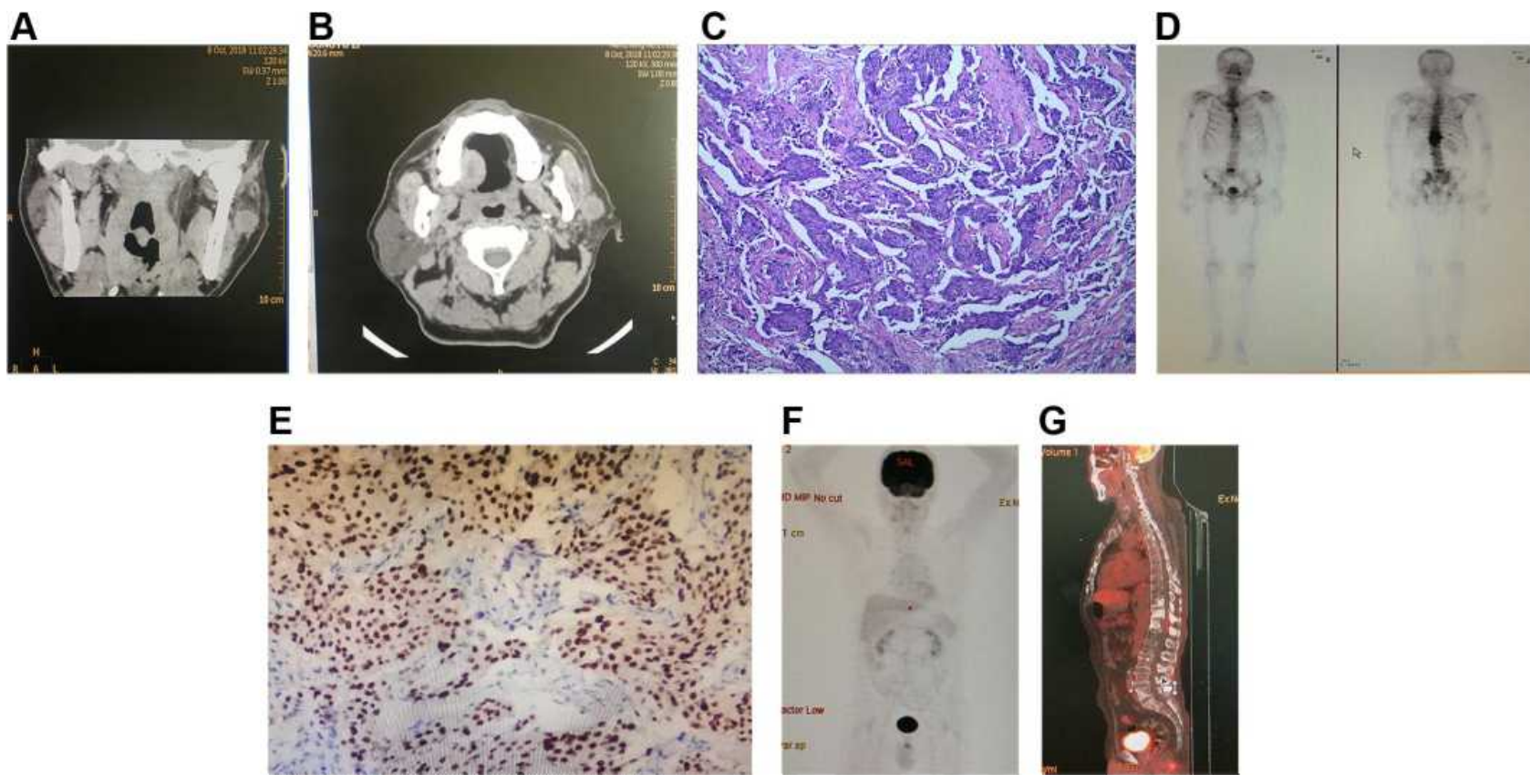

Figure I (A and B) Computer tomography showed a mass in the shallow lobe of the left parotid gland. (C) Post-operative pathology indicated that the patient had duct carcinoma of the parotid gland. (D) Emission computed tomography revealed multiple bone metastases involving the spine, ribs, and pelvis before androgen deprivation therapy. (E) Androgen receptor immunohistochemistry showed a strong positive reaction in tumour cells. (F and $\mathbf{G )}$ Positron emission tomography and computed tomography (PET-CT) showed no significant evidence of bone metastases after androgen deprivation therapy. 
involving the spine, ribs, and pelvis (Figure 1D). No other metastases were found in the neck, lungs or liver. The patient was diagnosed with bone metastasis of parotid gland cancer based on his previous medical history.

Subsequent treatment for the recurrent lesions included radiotherapy (40 Gy; 2 Gy once a day, five days a week) for the spine bone metastases due to bone pain and the risk for pathological fractures, and bisphosphonates (once every 4 weeks) to prevent bone destruction. While the patient was receiving treatment, his previous surgical specimens were taken for genetic testing, and the tumour was found to have mutations in HRAS (exon 3, p. Q61K) and PIK3CA (exon10, p.E545K). No gene amplification of HER-2 or mutations in other related genes were observed. In addition, immunohistochemistry showed strong positive staining for androgen receptor (AR) expression (Figure 1E). Finally, we chose androgen deprivation therapy that targeted the AR as a first-line therapy because no other drugs were available. The anti-AR endocrine therapy administered to the patient consisted of bicalutamide (50mg; once a day) and leuprolide (3.75mg; once every 4 weeks). Leuprolide was first administered one week after bicalutamide treatment; subsequently, the two drugs were administered in combination. After 7 months of combined androgen blockade (CAB) therapy, the patient's backache symptoms had resolved, positron emission tomography and computed tomography (PET-CT) showed no significant evidence of bone metastases (Figure 1F and G), and his serum CA724 levels had returned to near-normal levels. The patient is currently alive with a progression-free survival (PFS) of over 7 months to date.

Throughout the endocrine therapy, the patient reported that he tolerated the treatment well, did not experience any significant adverse events, and found it convenient to receive treatment in an outpatient setting.

\section{Discussion and Conclusion}

Human salivary glands comprise the parotid gland, submandibular gland, small salivary gland and sublingual glands. The parotid gland is affected in the majority of salivary gland carcinoma cases. ${ }^{1}$ SDC is a subtype of primary carcinoma ${ }^{2}$ and is defined as a rare and aggressive salivary gland malignancy with high rates of recurrence and metastasis. ${ }^{3}$ The age-standardized incidence of salivary gland carcinoma in Shanghai, China, is 7.99 per $1,000,000$ people per year, and the incidence did not change significantly from 2003 to 2012. ${ }^{4}$ Radiologically, SDC is often detected by CT or magnetic resonance imaging (MRI). Several features, such as ill-defined borders, infiltrative masses with frequent calcification, and necrosis in the primary tumour, can be seen on CT or MRI. ${ }^{5}$ Despite advances in imaging techniques, CT and MRI cannot reliably distinguish between benign and malignant lesions. Biopsies are needed to obtain histological information about the tumours before surgical treatment. Biopsy methods include core needle biopsy (CNB) and fine-needle aspiration (FNA). Schmidt et $\mathrm{al}^{6}$ conducted a systematic literature review including 277 cases and showed that $\mathrm{CNB}$ of salivary gland lesions is more sensitive and specific than FNA. The disadvantages of CNB include pain, haematoma and an increased risk of nerve damage. Additionally, the study suggested that FNA may be more useful than CNB for diagnosing lymph node metastases. The common sites of metastasis of salivary gland carcinoma are the lung (49\%), bones (40\%), liver (19\%) and other rare sites such as soft tissue $(9 \%){ }^{1}$ Metastasis to the cervical lymph nodes (19.6\%) and other distant sites are prognostic factors for risk. ${ }^{7}$ Many factors are associated with increased risk for lymph node metastasis, including older age, male sex, large tumour size, high-grade tumour, tumour extension and high-risk pathology. The number and ratio of positive lymph nodes are independent factors for patients with major salivary gland cancer. ${ }^{8}$ Treatments for SDC include surgery, radiotherapy, chemotherapy and targeted therapies, similar to the treatment options available for most other cancers. Single surgical primary tumour excision is the primary therapy for salivary gland carcinoma with negative lymph nodes of the neck, and complete neck dissection may be performed in patients with clinical or radiological neck involvement. Post-operative radiotherapy is often recommended for cases with the features of middle- or high-grade tumours, close or positive resection, perineural invasion, positive lymph nodes, advanced disease stage (T3-4), the presence of lymphatic or vascular infiltration and adenoid cystic carcinomas. 9,10 In addition, radical radiotherapy is used for unresectable or residual tumours. No standard treatment options are available for distant metastatic disease. Chemotherapy, a systemic therapy, is recommended by the NCCN (2020) for the palliative treatment of metastatic disease or for locoregional recurrence that cannot be treated with surgery or irradiation.

The androgen receptor was first identified in testicular extracts from pigs and dogs by Dr. Charles BrownSéquard in the 19th century. ${ }^{11}$ The human AR protein is a member of the nuclear receptor family and is 
approximately 919 amino acids in length; the length of the AR protein can vary due to polyglutamine and polyglycine stretches of variable length. ${ }^{12}$ The AR protein consists of 4 domains: an N-terminal domain, a DNA-binding domain, a hinge domain, and a ligand-binding domain. ${ }^{13}$ The ligand-binding domain is unbound from heat shock protein in the presence of the AR receptor ligand, and the AR translocates into the nucleus from the cytoplasm to act as a nuclear transcription factor. In humans, androgen- and AR-mediated signalling pathways play important roles in regulating the transcription of multiple effector genes that can increase cell growth, differentiation and survival. These signalling pathways control the development of the male phenotype and the maintenance of reproductive functions and other parameters, such as muscle mass. Androgen- and AR-mediated signalling pathways are associated with several hormone-related diseases, including prostate cancer and breast cancer, along with other signalling pathways, including the PI3K/Akt/mTOR and MAPK pathways. ${ }^{14}$ The AR is physiologically widely expressed in many human tissues. ${ }^{15}$ Several studies have shown that the frequency of AR immunopositivity in SDC varies between $69.4 \%$ and $94 \%$ (177/188 in 188 samples from 163 patients), including in 140/148 (94\%), 11/11 (100\%) regional lymph node metastases and 26/29 (90\%) distant metastases; however, the frequency of AR immunopositivity is approximately $16 \%$ in the non-SDC salivary gland carcinoma group. ${ }^{16,17}$ It might be possible to use ARtargeted treatment strategies for AR-positive tumours, and this ADT treatment might be effective for patients with SDC. In 2003, Locati et al ${ }^{18}$ reported the first case of AR-positive relapsed parotid adenocarcinoma treated with ADT and reported that the patient treated with $\mathrm{CAB}$ (triptorelin and bicalutamide) achieved a complete response; however, the patient ultimately died of chronic myelocytic leukaemia. ElineB ${ }^{19}$ described 35 patients with advanced AR-positive SDC who received first-line ADT (28 patients received bicalutamide and 7 patients received an LHRH analogue and low-dose bicalutamide). Six patients had a partial response (18\%), and 11 had stable disease $(32 \%)$, leading to a clinical benefit ratio of $50 \%$. The median PFS for the ADT-treated patients was 4 months, and the median duration of clinical benefit was 11 months. The median overall survival (OS) was 17 months. Another study showed that patients treated with ADT (CAB; bicalutamide plus triptorelin) had a better overall response rate (ORR; 64.7\%) than those who did not receive the treatment, a median PFS of 11 months and a median OS of 44 months. $^{20}$ In addition, a prospective Phase II study showed that CAB had equivalent efficacy to and less toxicity than other treatments for patients with AR-positive metastatic or locally advanced unresectable salivary gland carcinoma. ${ }^{21}$ ADT results in a similar OS as achieved with conventional cytotoxic chemotherapy when used as a first-line treatment, but response rates in patients treated with ADT are higher than in those treated with first-line chemotherapy. ${ }^{22} \mathrm{CAB}$ may be more effective for patients with AR-positive SDC than single-agent AR antagonists such as bicalutamide. CYP17A inhibitors, such as abiraterone acetate, may be suitable for patients resistant to conventional $\mathrm{ADT}^{23}$ The mechanisms of resistance to AR inhibitors in SDC were not defined. However, some mechanisms of resistance to AR inhibitors have been identified in prostate cancer. Point mutations within the AR ligand-binding domain, signals activated by a related kinase not targeted by AR inhibitors and complete AR independence could lead to resistance to AR inhibitors. ${ }^{24}$ A retrospective clinical trial aimed towards reducing the recurrence of stage $4 \mathrm{a} / \mathrm{b}$ disease confirmed that patients with poor-risk, AR-positive SDC who received adjuvant ADT after tumour resection had a significantly longer disease-free survival (DFS) than the control group (3-year DFS 48.2\% VS 27.7\%, median DFS 33 months VS 21 months). ${ }^{25}$ A combination of endocrine therapy and chemoradiotherapy may increase efficacy for patients with SDC, similar to the benefits observed following the use of this treatment in prostate cancer. Several case reports have shown that ADT plus chemotherapy or radiotherapy is suitable for patients with AR-positive SDC that is considered unresectable or for patients who refuse surgery. ${ }^{26,27}$

To our knowledge, this is the first case described with elevated CA724 and there are very few cases with metastatic salivary duct carcinoma treated with combined radiotherapy and endocrine therapy. Previous studies have demonstrated that ADT is an important and effective therapeutic approach for patients with AR-positive salivary gland carcinoma. There are still some shortcomings regarding the use of ADT. First, few studies involving a large patient sample size have been performed. Second, the mechanisms of resistance to conventional ADT should be focused on in SDC. Further more, predictive biomarkers, different drugs, drug dosages, and combinations with other treatments need to be explored in future studies in the context of ADT efficacy. We believe that ADT will become increasingly standardized as a first-line treatment for AR-positive SDC in the near future. 


\section{Ethics Approval and Consent for Publication}

This research was approved by the research ethics committee of The First Hospital of Nanchang, and written informed consent for publication of the clinical details and images was obtained from the patient.

\section{Acknowledgments}

We thank Dr.Xuchang Tao for providing the radiological images presented in this article. We also thank all the clinicians for their contribution to the treatment of the patient.

\section{Disclosure}

The authors report no conflicts of interest.

\section{References}

1. Safina A, Robert B, Frank LP, et al. Distant metastases in patients with carcinoma of the major salivary glands. Ann Surg Oncol. 2015;22(12):4014-4019. doi:10.1245/s10434-015-4454-y

2. Masato N, Yuichiro T, William CF, et al. Salivary duct carcinoma: updatesin histology, cytology, molecular biology, and treatment. Cancer Cytopathol. 2020;128(10):693-703. doi:10.1002/cncy.22288

3. Takahashi H, Tada Y, Saotome T, et al. Phase II trial of trastuzumab and docetaxel in patients with human epidermal growth factor receptor 2-positive salivary duct carcinoma. J Clin Oncol. 2019;37 (2):125-134. doi:10.1200/JCO.18.00545

4. Fu JY, Wu CX, Shen SK, et al. Salivary gland carcinoma in Shanghai (2003-2012): an epidemiological study of incidence, site and pathology. BMC Cancer. 2019;19(1):350. doi:10.1186/s12885-019-5564-x

5. Weon YC, Park SW, Kim HJ, et al. Salivary duct carcinomas: clinicaland $\mathrm{CT}$ and MR imaging features in 20 patients. Neuroradiology. 2012;54(6):631-640. doi:10.1007/s00234-012-1014-Z

6. Schmidt RL, Hall BJ, Layfield LJ. A systematic review and meta-analysis of the diagnostic accuracy of ultrasound-guided core needle biopsy for salivary gland lesions. Am J Clin Pathol. 2011;136 (4):516-526. doi:10.1309/AJCP5LTQ4RVOQAIT

7. Nam SJ, Roh JL, Cho KJ, et al. Risk factors and survival associated with distant metastasis in patients with carcinoma of the salivary gland. Ann Surg Oncol. 2016;23(13):4376-4383. doi:10.1245/ s10434-016-5356-3

8. Qian K, Sun W, Guo K, et al. The number and ratio of positive lymph nodes are independent prognostic factors for patients with major salivary gland cancer: results from the surveillance, epidemiology, and End Results dataset. Eur J Surg Oncol. 2019;45(6):1025-1032. doi:10.1016/j.ejso.2018.11.008

9. Storey MR, Garden AS, Morrison WH, et al. Postoperative radiotherapy for malignant tumors of the submandibular gland. Int J Radiat Oncol Biol Phys. 2001;51(4):952-958. doi:10.1016/ s0360-3016(01)01724-2

10. Safdieh J, Givi B, Osborn V, et al. Impact of adjuvant radiotherapy for malignant salivary gland tumors. Otolaryngol Head Neck Surg. 2017;157(6):988-994. doi:10.1177/0194599817717661

11. Brown-Sequard CE. Note on the e_ects produced on man by subcutaneous injections of a liquid obtained from the testicles of animals. Lancet. 1889;134:105-107. doi:10.1016/S0140-6736(00) 64118-1
12. Claessens F, Denayer S, Tilborgh NV, et al. Diverse roles of androgen receptor (AR) domains in AR-mediated signaling. Nucl Recept Signal. 2008;6:e008. doi:10.1621/nrs.06008

13. Tan MH, Li J, Xu HE, et al. Androgen receptor: structure, role in prostate cancer and drug discovery. Acta Pharmacol Sin. 2015;36 (1):3-23. doi:10.1038/aps.2014.18

14. Sarker D, Reid AH, Yap TA, et al. Targeting the PI3K/AKT pathway for the treatment of prostate cancer. Clin Cancer Res. 2009;15 (15):4799-4805. doi:10.1158/1078-0432

15. Ruizeveld de Winter JA, Trapman J, Vermey M, et al. Androgen receptor expression in human tissues: an immunohistochemical study. $J$ Histochem Cytochem. 1991;39(7):927-936. doi:10.1177/ 39.7.1865110

16. Xu B, Dogan S, Rasheed MRHA, et al. Androgen receptor immunohistochemistry in salivary duct carcinoma: a retrospective study of 188 cases focusing on tumoral heterogeneity and temporal concordance. Hum Pathol. 2019;93:30-36. doi:10.1016/j. humpath.2019.08.007

17. Liang L, Williams MD, Bell D. Expression of PTEN, Androgen Receptor, HER2/neu, Cytokeratin 5/6, Estrogen Receptor-Beta, HMGA2, and PLAG1 in salivary duct carcinoma. Head Neck Pathol. 2019;13(4):529-534. doi:10.1007/s12105-018-0984-5

18. Locati LD, Quattrone P, Bossi P, et al. A complete remission with androgen-deprivation therapy in a recurrent androgen receptor-expressing adenocarcinoma of the parotid gland. Ann Oncol. 2003;14(8):1327-1328. doi:10.1093/annonc/mdg331

19. Boon E, Boxtel W, Buter J, et al. Androgen deprivation therapy for androgen receptor-positive advanced salivary duct carcinoma: a nationwide case series of 35 patients in The Netherlands.Head. Neck. 2018;40(3):605-613. doi:10.1002/hed.25035

20. Locati LD, Perrone F, Cortelazzi B, et al. Clinical activity of androgen deprivation therapy in patients with metastatic/relapsed androgen receptor-positive salivary gland cancers. Head Neck. 2016;38 (5):724-731. doi:10.1002/hed.23940

21. Fushimi C, Tada Y, Takahashi H, et al. A prospective phase II study of combined androgen blockade in patients with androgen receptor-positive metastatic or locally advanced unresectable salivary gland carcinoma. Ann Oncol. 2018;29(4):979-984. doi:10.1093/ annonc/mdx 771

22. Viscuse PV, Price KA, Garcia JJ, et al. First line androgen deprivation therapy vs. chemotherapy for patients with androgen receptor positive recurrent or metastatic salivary gland carcinoma-A Retrospective Study. Front Oncol. 2019;9:701. doi:10.3389/fonc.2019.00701

23. Urban D, Rischin D, Angel C, et al. Abiraterone in metastatic salivary duct carcinoma. J Natl Compr Canc Netw. 2015;13 (3):288-290. doi:10.6004/jnccn.2015.0040

24. Watson PA, Arora VK, Sawyers CL. Emerging mechanisms of resistance to androgen receptorinhibitors in prostate cancer. Nat Rev Cancer. 2015;15(12):701-711. doi:10.1038/nrc4016

25. Boxtel WA, Locati LD, Grunsven A, et al. Adjuvant androgen deprivation therapy for poor-risk, androgen receptor-positive salivary duct carcinoma. Eur J Cancer. 2019;110:62-70. doi:10.1016/j. ejca.2018.12.035

26. Kuroda H, Sakurai T, Yamada M, et al. Effective treatment by both anti-androgen therapy and chemotherapy for a patient with advanced salivary duct carcinoma. Gan to Kagaku Ryoho. 2011;38(4):627-630.

27. Soper MS, Iganej S, Thompson LDR. Definitive treatment of androgen receptor-positive salivary duct carcinoma with androgen deprivation therapy and external beam radiotherapy. Head Neck. 2014;36(1): E4-7. doi:10.1002/hed.23383 


\section{Publish your work in this journal}

OncoTargets and Therapy is an international, peer-reviewed, open access journal focusing on the pathological basis of all cancers, potential targets for therapy and treatment protocols employed to improve the management of cancer patients. The journal also focuses on the impact of management programs and new therapeutic agents and protocols on patient perspectives such as quality of life, adherence and satisfaction. The manuscript management system is completely online and includes a very quick and fair peer-review system, which is all easy to use. Visit http://www.dovepress.com/ testimonials.php to read real quotes from published authors. 